\title{
Demora en la búsqueda de atención médica en pacientes adictos a sustancias psicoactivas
}

\section{Delay in seeking medical care in patients addicted to psychoactive substances}

\author{
Rubén Eliseo Valle Rivadeneyra*; Alberto Perales \\ CABRERA ${ }^{*, * *}$
}

* Facultad de Medicina de la Universidad Nacional Mayor de San Marcos. Lima, Perú.

** Director del Instituto de Ética en Salud de la Universidad Nacional Mayor de San Marcos. Lima, Perú.

Enviar correspondencia a:

Rubén Eliseo Valle Rivadeneyra.

Jr. Filadelfia N 2365. San Martín de Porres. Lima 31, Perú

E-mail: ruben_vr12@hotmail.com.

\section{RESUMEN}

Introducción: En la práctica clinica se observa que los pacientes adictos a sustancias psicoactivas acuden a los servicios de salud después de un largo periodo de tiempo y que los familiares conociendo del consumo no realizan una búsqueda temprana de la atención médica. Objetivos: Determinar el tiempo transcurrido desde que los pacientes empiezan el consumo hasta que buscan por primera vez atención médica especializada (TC) y el tiempo transcurrido desde que los familiares perciben el consumo hasta que buscan por primera vez atención médica especializada (TSAM). Determinar los factores que se asocian a una búsqueda temprana de la atención médica. Diseño: Estudio observacional, descriptivo de corte transversal. Se aplicó una encuesta estructurada validada por juicio de expertos en el área de adicciones a 113 familiares responsables de pacientes adictos a sustancias psicoactivas de 5 hospitales de la ciudad de Lima. Resultados: Los pacientes acuden a la atención médica especializada por primera vez después de 11.22 años de iniciado el consumo y los familiares tardan en buscar atención médica 4.69 años desde que perciben el consumo. El género femenino, la concepción de la adicción como enfermedad y no esperar la decisión del paciente para acudir a la atención médica, reducen el TC y TSAM en la mayoria de sustancias. Conclusiones: Los pacientes adictos a sustancias psicoactivas acuden a los servicios de salud después de un TC y TSAM prolongados y la demora en buscar atención médica por los familiares ocasiona que los pacientes continúen consumiendo sustancias.

Palabras clave: Trastornos Relacionados con Sustancias, Accesibilidad a los Servicios de Salud, Diagnóstico Temprano, Familia.

\section{ABSTRACT}

Introduction: Clinical practice shows that addict patients tend to seek attention from health services after a long period of time, and that their relatives do not seek early medical care, despite knowledge of the addiction. Objectives: To determine the time elapsed between the beginning of patients' substance use and when they first seek specialized medical care (TC) and the time elapsed between relatives perceiving the substance use and when they first seek specialized medical care (TSAM); also, to identify the factors associated with seeking medical care earlier. Design: Observational, descriptive study. A structured survey, validated by experts in the field of addiction, was applied to 113 family members responsible for patients addicted to psychoactive substances in 5 hospitals in the city of Lima. Results: Patients seek specialist medical care for the first time 11.22 years after commencement of substance use, and family members take to 4.69 years to seek medical care from when they perceive of the substance use. Female gender, the concept of addiction as a disease and not waiting for the patient's decision before seeking medical care reduce TC and TSAM in the case of most substances. Conclusions: Patients addicted to psychoactive substances turn to health services only after long TC and TSAM; moreover, the delay in seeking medical care on the part of relatives means that patients continue to use substances.

Key words: Substance-Related Disorders, Accessibility to Health Services, Early Diagnosis, Family. 


\section{INTRODUCCIÓN}

E consumo de drogas es un problema que ha tomado gran relevancia en el mundo por el impacto económico, social y de salud que produce'. En el año 2007, se estimó que 200 millones de personas consumían drogas a nivel mundial ( $4.8 \%$ de la población entre 15 y 64 años) $)^{2}$ y aunque la mayoría de sustancias se consume en Norteamérica y Europa, cada vez está aumentando el número de usuarios en países asiáticos y sudamericanos ${ }^{3}$. El Perú es uno de los países de mayor producción a nivel mundial de cocaína junto a Colombia y Bolivia y desde el 2005 ha registrado un aumento del $7 \%$ en su producción ${ }^{2,4}$.

En el Perú y en otras regiones del mundo ${ }^{5}$, el consumo de sustancias es un problema de salud pública y por la envergadura del problema el Estado Peruano en el Plan Nacional Concertado de Salud 2007-2011 reconoce a la adicción como uno de los principales problemas sanitarios ${ }^{6}$; además, hasta el año 20067.156 .081 y 104.038 personas habian consumido sustancias legales e ilegales en el último año, respectivamente. El alcohol es la sustancia legal de mayor consumo con una prevalencia anual de 67\%. La sustancia ilegal más consumida es la marihuana con una prevalencia anual de $0.7 \%$, seguida de la pasta básica de cocaína (PBC) (0.3\%) y cocaína $(0.3 \%)^{7}$.

El consumo de sustancias produce consecuencias negativas a nivel biológico, psicológico y social, con serios problemas familiares ${ }^{8}$. Durante el transcurso de la adicción, la familia del adicto experimenta elevados niveles de estrés, violencia ${ }^{9}$, sintomas de ansiedad y depresión ${ }^{10,11}$. La presencia de un problema de adicción constituye el reflejo de los desajustes del sistema familiar; por ejemplo, muchos de los problemas de alcohol y uso de drogas durante la adolescencia pueden ser el resultado de problemas que se han venido dando desde la infancia ${ }^{12}$ y que se ven reflejados en actos desde tempranas edades como robo en la escuela y daño a la propiedad ${ }^{13}$. También muchos de los consumidores han sido víctimas de abuso sexual, físico y emocional por parte de sus familiares ${ }^{14}$. Por lo tanto, por los problemas que ocasiona el adicto dentro de la familia y por la presencia de algunas condiciones familiares patológicas que favorecen el desarrollo de la adicción, la familia no sólo resulta un componente esencial en el diagnóstico y tratamiento sino que frecuentemente necesita tratamiento al igual que la persona adicta ${ }^{15}$.

Los problemas relacionados a las adicciones son complejos y pueden persistir por varios años ${ }^{16}$; además sólo una proporción de las personas que consumen sustancias llegan a utilizar los recursos sanitarios. Un importante primer paso en el tratamiento de los desordenes mentales y de las adicciones se asocia al contacto temprano del paciente con los servicios de salud ${ }^{5,17}$ para prevenir las consecuencias del consumo crónico; sin embargo, se han identificado diferentes factores que impiden el acceso a la salud tales como, las barreras económicas ${ }^{18}$, geográficas ${ }^{19}$, el género ${ }^{20}$, la edad ${ }^{17,21}$ y factores propios de los servicios de salud como la no adecuación a los programas de tratamiento ${ }^{16}$ y la falta del tiempo del personal ${ }^{22}$. Nuño-Gutiérrez, señala que la concepción de adicción como "vicio social" provoca reacciones afectivas que interfieren con la utilización de los servicios de salud ${ }^{23}$.

Una particularidad de estos pacientes es la ausencia de conciencia de enfermedad ${ }^{24,25}$, característica clínica que contribuye a que no busquen atención médica oportuna y ocasiona una demora en la atención médica en aproximadamente 10 años ${ }^{5,26}$; sumado a esto, se encuentra la escasa motivación que presentan para recibir tratamiento ${ }^{25}$. Por las características descritas es muy difícil que el adicto acuda por voluntad propia a la atención médica y es en estas circunstancia donde cobra importancia el rol de la familia en la identificación del problema y en la búsqueda de la atención médica; sin embargo, en la práctica médica se observa que muchos familiares conociendo del consumo de sustancias por parte del paciente, no buscan una atención médica de forma oportuna y más bien conviven con la adicción en el seno familiar e incluso muchos de ellos describen un estado de coadicción con el adicto ${ }^{10}$.

Sobre la base de lo descrito, el objetivo del presente trabajo se orienta a evaluar estas conductas pasivas observadas en los pacientes y familiares. Primero en los pacientes, a través del tiempo de consumo (TC) establecido por el tiempo transcurrido desde que inicia el consumo de sustancias hasta que llega por primera vez a la atención médica especializada (psiquiatra) y segundo de los familiares, a través del tiempo sin atención médica (TSAM) establecido por el tiempo transcurrido desde que los familiares perciben el consumo de sustancias psicoactivas hasta que buscan por primera vez atención médica especializada. Así mismo, se evalúan los factores sociodemográficos y culturales que se asocian a una búsqueda de atención médica más temprana.

\section{METODOLOGÍA}

Estudio analítico, observacional de corte transversal. La población de estudio estuvo conformada por los familiares responsables de los pacientes consumidores de sustancias que se encuentren o hayan sido hospitalizados en algún centro hospitalario y que actualmente asisten a la terapia grupal familiar en los Servicios de Adicciones de los Hospitales Nacionales de la ciudad de Lima. Los establecimientos de salud donde se llevó a cabo la evaluación fueron: Instituto Nacional de Salud Mental Honorio Delgado-Hideyo Noguchi, Hospital Hermilio Valdizán, Hospital Nacional Guillermo Almenara Irigoyen, Hospital Edgardo Rebagliatti Martins y Hospital Victor Larco Herrera, que representan a los 5 centros especializados donde se brinda atención asistencial por esta patología en la ciudad de Lima.

Criterios de inclusión: Familiares de pacientes que consumen sustancias psicoactivas, familiares que asisten regularmente a la terapia grupal familiar, familiares de pacientes que se encuentren o hayan estado internados en el servicio de adicciones. Criterios de exclusión: Familiares de pacientes con un problema de adicción diferente al consumo de sustancias psicoactivas y los familiares de pacientes con algún trastorno psiquiátrico diferente a los Trastornos relacionados con sustancias. 
La evaluación se realizó a través de una encuesta estructurada, conformada de 3 secciones: Datos sociodemográficos (Familiar: Edad, género, grado de instrucción, parentesco con el paciente; Paciente: Edad, edad de inicio de consumo, grado de instrucción, sustancia consumida); mediciones de tiempos (TC y TSAM) y factores asociados (decisión, ocupación, conocimiento, primera institución donde realizó la búsqueda de ayuda). La encuesta fue validada por medio de juicio de expertos, especialistas en el área de las adicciones, para luego llevar a cabo una prueba piloto en 10 familiares, en el Instituto Nacional de Salud Mental Honorio Delgado-Hideyo Noguchi. La recolección de datos se realizó el dia en que los familiares acudieron a las sesiones familiares en cada una de las instituciones. Se aplicó la encuesta previo consentimiento de los familiares sin solicitar la identidad a los participantes, registrando cada encuesta con un número para cumplir con los criterios de confidencialidad.

Se realizó un análisis exploratorio, analizando los patrones de frecuencia en las variables cualitativas y medida de tendencia central en caso de las variables cuantitativas. El análisis de distribución normal se realizó a través del histograma y la prueba de Kolmorogov-Smirnov. El análisis univariado se realizó a través de la presentación de figuras y tablas de frecuencia. En el análisis bivariado se aplicó la regresión y correlación lineal de Pearson para estudiar la asociación entre las variables cuantitativas. La asociación entre variables cuantitativas y factores sociodemográficos se hizo a través de pruebas no paramétricas: Prueba de MannWhitney y Kruskal-Wallis. Se trabajó con un nivel de confianza del $95 \%$ y un nivel de significancia de 0.05. Se utilizó el paquete estadistico SPSS v 13.0.

\section{RESULTADOS}

Se encuestaron 119 familiares responsables de pacientes adictos a sustancias psicoactivas de 5 hospitales de la ciudad de Lima, 6 encuestas fueron anuladas: 4 por estar incompletas y 2 por ser ininteligibles, por lo que el análisis final se realizó en base a 113 encuestas.

Las características sociodemográficas de los pacientes y familiares responsables se observan en la tabla 1.

El $62.8 \%$ de los familiares eran padres de los pacientes y el $18.6 \%$ eran los cónyuges, una baja proporción estuvo constituida por los hermanos e hijos de los pacientes. Sólo el $19.5 \%$ busco en primera instancia una atención médica psiquiátrica, mientras que el $29.2 \%$ acudió a una comunidad terapéutica, el porcentaje restante que constituyó la mayoría (51.3\%) buscó atención en profesionales no médicos, religión y amigos. El $70.8 \%$ de la población estudiada consumia más de una sustancia al momento de la evaluación, representando la gran mayoría (41.6\%) aquellos que consumian 2 sustancias. El alcohol es consumido por el $61 \%$ de pacientes, seguido por un $43 \%$ que consume marihuana, el consumo de PBC resultó ser mayor que el de la cocaina ( $41 \%$ y $37 \%$, respectivamente). La sustancia menos consumida fue el tabaco con el $22 \%$ de consumidores.
Tabla 1. Características sociodemográficas de los familiares responsables y de los pacientes adictos a sustancias psicoactivas. Lima - 2008

\begin{tabular}{|c|c|c|c|c|}
\hline & \multicolumn{2}{|c|}{ Familar resognsable } & \multicolumn{2}{|c|}{ Paccente } \\
\hline & h & $*$ & ก & $*$ \\
\hline \multicolumn{5}{|l|}{ Cinuteo } \\
\hline Femenino & 84 & 74.34 & 13 & 11.50 \\
\hline Masculino & 29 & 25.65 & 100 & 68.50 \\
\hline \multicolumn{5}{|c|}{ Grado de instruction } \\
\hline Primaria & 16 & 14.16 & 2 & 1.77 \\
\hline Secandaria & 46 & 40.71 & 75 & 66.37 \\
\hline Superior & 51 & 45.13 & 36 & $31.6 \%$ \\
\hline \multicolumn{5}{|l|}{ Edad } \\
\hline $16-30$ & 2 & 1.77 & 57 & 50.44 \\
\hline $31-45$ & 20 & 17.70 & 25 & 22.12 \\
\hline $46-60$ & 55 & 48.67 & 23 & 20.35 \\
\hline $61-80$ & 36 & 31.86 & 8 & 7.06 \\
\hline \multicolumn{5}{|c|}{ Edad de inicio del consumo } \\
\hline 10.15 & & & 43 & 36.13 \\
\hline $16-20$ & & & 48 & 40.34 \\
\hline 21.25 & & & 10 & 8.40 \\
\hline 226 & & & 12 & 10.08 \\
\hline
\end{tabular}

Entre las características de la población se encuentra que la mayor proporción (83\%) desarrollaba alguna ocupación como estudiar o trabajar y el $17 \%$ no se encontraba desempeñando ninguna actividad académica o laboral. Sólo en el $21 \%$ de casos la decisión de asistir a la atención médica contó con la participación o del consentimiento del paciente, mientras que en el porcentaje restante la decisión fue tomada en forma autónoma por los familiares. Asimismo, sólo el $19 \%$ de familiares consideraba a la adicción como una enfermedad; mientras que el $81 \%$ la percibía como un "vicio social" durante el tiempo de consumo.

Al analizar el tiempo que demoran los pacientes adictos a sustancias psicoactivas en llegar a la atención médica especializada (TC); se observa que el TC en forma general (todas las sustancias) es de 11.22 años y al analizar el TC según cada sustancia, se observa que el alcohol presenta el mayor TC (14 años), seguido de los consumidores de tabaco (13.9 años); el menor TC es para aquellos pacientes que consumen marihuana (Fig.1). Al ser agrupadas las sustancias según su categoria legal, se observa que los pacientes que consumen sustancias legales(alcohol y tabaco) demoran más tiempo en llegar a la atención médica ( $\mathrm{TC}=15.9$ años) en comparación con los pacientes consumidores de sustancias ilegales ( $\mathrm{TC}=7.1$ años) y los que consumen sustancias legales e ilegales, (consumo mixto) que es 12.8 años.

El tiempo transcurrido desde que los familiares perciben el consumo hasta que buscan atención médica especializada (TSAM), en forma general es de 4.69 años. El mayor TSAM es para aquellos familiares cuyos pacientes consumen PBC (8.4 años), seguido por el TSAM de los consumidores de tabaco (6.2 años), el menor TSAM es para aquellos familiares cuyos pacientes consumen marihuana (2.7 años). Según la categoria legal, los familiares de pacientes que consumen sustancias legales (6.2 años) demoran más en buscar atención médica en comparación con aquellos que consumen sustancias ilegales (3.9 años) y de aquellos que presentan un consumo mixto de sustancias (5.7 años). 


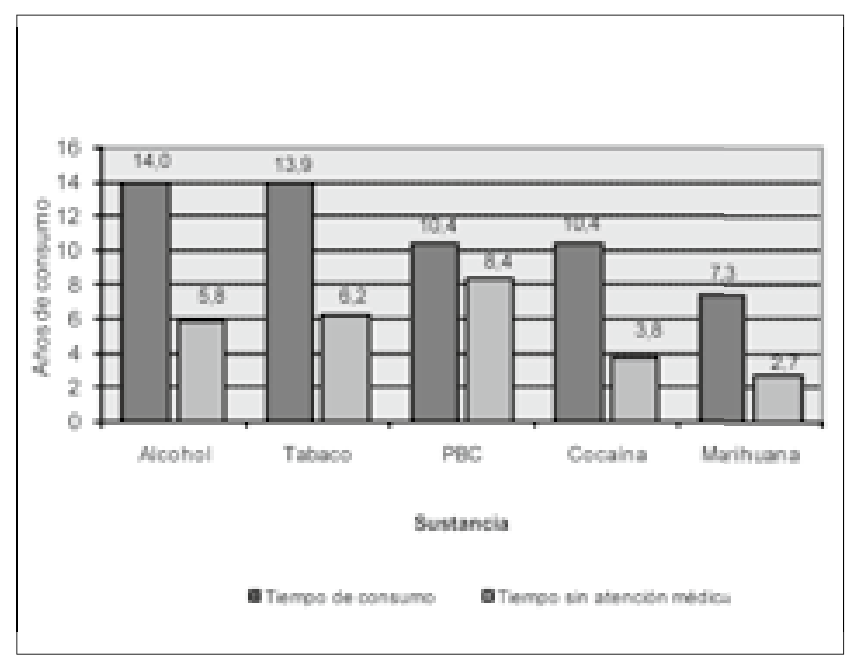

Figura 1. Tiempo de consumo y Tiempo sin atención médica en pacientes adictos a sustancias psicoactivas. Lima - 2008

La relación entre el TC y el TSAM es directamente proporcional, es decir un mayor TSAM se asocia a un mayor TC (Fig. 2). El coeficiente de determinación $\left(R^{2}\right)$ es de 0.45 lo que significa que el $45 \%$ de la variación del TC es explicado por el modelo de regresión y el Coeficiente de correlación de Pearson $(R)$ es de 0.67 lo que nos muestra que ambas variables se relacionan en una intensidad moderada.

La asociación entre el TC y los factores asociados se observan en la tabla 2. La decisión de asistir a la atención médica se encontró asociada significativamente al TC en los casos de todas las sustancias evaluadas, lo que muestra que cuando la decisión de asistir a la atención médica es tomada sin la participación del paciente el TC resulta menor en comparación que si se espera de la participación o de la aceptación del paciente para asistir a la atención médica. El género también se encontró asociado al TC en el caso de los consumidores de alcohol

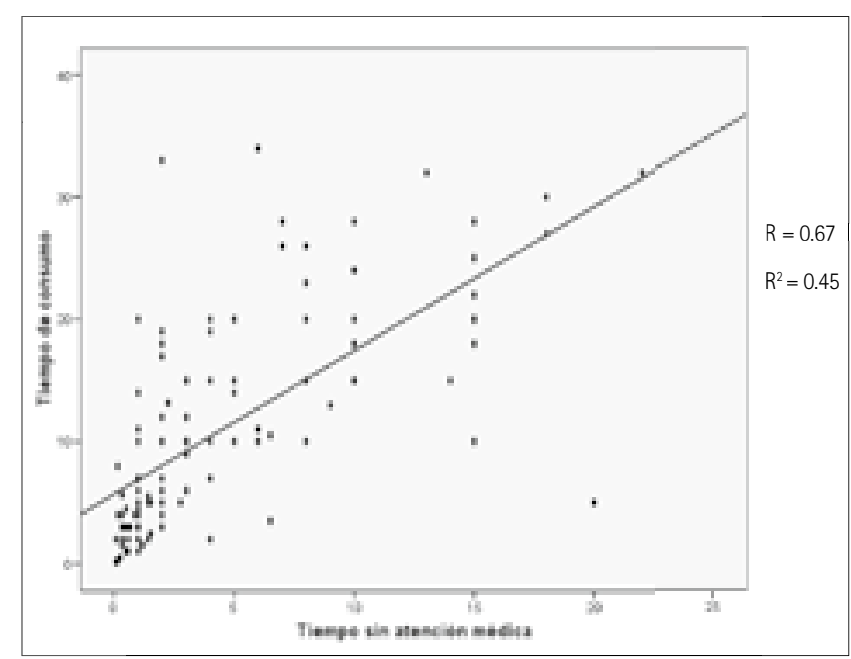

Figura 2. Relación entre el Tiempo de consumo de consumo y el Tiempo sin atención médica en pacientes adictos a sustancias psicoactivas. Lima - 2008.

y marihuana, observándose que las mujeres consumidoras de alcohol llegan más rápido a la atención médica en comparación con los varones (mujeres $=8.4$, varones $=14.9$ años), el caso contrario ocurre entre los consumidores de marihuana (mujeres $=10.7$, varones $=6.8$ años).

Al analizar la relación entre el TSAM y los factores asociados (Tabla 3), se observa que el conocimiento acerca del consumo de sustancias psicoactivas por parte de los familiares se encuentra asociado significativamente al TSAM en los casos de los consumidores de alcohol y $\mathrm{PBC}$, lo que significa que aquellos familiares que conciben a la adicción como una enfermedad demoran menos tiempo en buscar atención médica en comparación a aquellos que la conciben como un "vicio social". La decisión de asistir a la atención médica también se encuentra asociada significativamente al TSAM en los casos de todas las sustancias evaluadas a excepción de la cocaína.

Tabla 2. Factores relacionados al tiempo de consumo en pacientes adictos a sustancias psicoactivas. Lima 2088.

\begin{tabular}{|c|c|c|c|c|c|c|c|c|c|c|}
\hline \multirow[t]{2}{*}{ Sustancia } & \multicolumn{2}{|c|}{ Alcohol } & \multicolumn{2}{|c|}{ Tabaco } & \multicolumn{2}{|c|}{ PBC } & \multicolumn{2}{|c|}{ Cocaína } & \multicolumn{2}{|c|}{ Marihuana } \\
\hline & Media(DE) & $p$ & Media(DE) & $p$ & Media(DE) & $p$ & Media(DE) & $p$ & Media(DE) & $p$ \\
\hline Grado de instrucción & & 0.09 & & 0.43 & & 0.13 & & 0.35 & & 0.67 \\
\hline Primaria & $30.0(2.8)$ & & $30.0(2.8)$ & & & & & & & \\
\hline Secundaria & $12.9(8.3)$ & & $11.4(7.1)$ & & $9.3(7.8)$ & & $9.3(7.5)$ & & $6.7(6.4)$ & \\
\hline Superior & $14.5(9.8)$ & & $15.6(8.8)$ & & $13.7(9.7)$ & & $11.6(8.8)$ & & $8.6(9.0)$ & \\
\hline Género & & 0.034 & & 0.49 & & 0.69 & & 0.31 & & 0.03 \\
\hline Femenino & $8.4(6.7)$ & & $10.8(6.8)$ & & $7.3(4.6)$ & & $7.8(7.7)$ & & $10.7(6.7)$ & \\
\hline Masculino & $14.9(9.2)$ & & $14.5(9.1)$ & & $10.6(8.6)$ & & $11.1(8.6)$ & & $6.8(7.3)$ & \\
\hline Ocupación & & 0.66 & & 0.68 & & 0.8 & & 0.22 & & 0.68 \\
\hline $\mathrm{Si}$ & $13.8(9.4)$ & & $13.5(9.0)$ & & $10.3(8.4)$ & & $9.8(8.2)$ & & $7.3(7.6)$ & \\
\hline No & $14.8(7.9)$ & & $15.3(8.6)$ & & $10.9(8.9)$ & & $13.7(9.4)$ & & $7.6(6.9)$ & \\
\hline Decisión & & 0.0002 & & 0.03 & & 0.0001 & & 0.02 & & 0.02 \\
\hline Sin participación del paciente & $11.4(8.2)$ & & $11.6(8.9)$ & & $8.3(7.0)$ & & $9.3(7.8)$ & & $6.6(7.0)$ & \\
\hline Con participación del paciente & $20.8(8.1)$ & & $18.8(6.6)$ & & $20.7(7.4)$ & & $19.3(8.6)$ & & $13.5(7.8)$ & \\
\hline
\end{tabular}

Asociación estadísticamente significativa $(\mathrm{p}<0.05)$.

La media está expresada en años.

DE: Desviación Estándar 
Tabla 3. Factores relacionados al Tiempo sin atención médica en pacientes adictos a sustancias psicoactivas. Lima-2008.

\begin{tabular}{|c|c|c|c|c|c|c|c|c|c|c|}
\hline \multirow[t]{2}{*}{ Sustancia } & \multicolumn{2}{|c|}{ Alcohol } & \multicolumn{2}{|c|}{ Tabaco } & \multicolumn{2}{|c|}{ PBC } & \multicolumn{2}{|c|}{ Cocaína } & \multicolumn{2}{|c|}{ Marihuana } \\
\hline & Media(DE) & $p$ & Media(DE) & $p$ & Media(DE) & $p$ & Media(DE) & $p$ & Media(DE) & $p$ \\
\hline $\begin{array}{l}\text { Grado de instrucción del familiar } \\
\text { responsable }\end{array}$ & & 0.7 & & 0.53 & & 0.25 & & 0.17 & & 0.74 \\
\hline Primaria o ninguno & $6.1(5.6)$ & & $4.8(4.7)$ & & $6.3(7.4)$ & & $7.2(6.0)$ & & $3.0(2.9)$ & \\
\hline Secundaria & $6.0(4.7)$ & & 7.3(5.1) & & $4.0(5.5)$ & & $3.4(3.9)$ & & $2.9(4.0)$ & \\
\hline Superior & $5.6(5.8)$ & & $6.0(5.8)$ & & $4.8(5.3)$ & & $2.8(2.5)$ & & $2.4(3.0)$ & \\
\hline Género & & 0.07 & & 0.85 & & 0.98 & & 0.95 & & 0.13 \\
\hline Femenino & $2.6(1.4)$ & & 4.1(1.6) & & $2.3(1.1)$ & & $2.9(2.1)$ & & $2.9(1.5)$ & \\
\hline Masculino & $6.4(5.5)$ & & $6.5(5.6)$ & & $4.9(5.8)$ & & $3.9(4.3)$ & & $2.6(3.6)$ & \\
\hline Decisión & & 0.001 & & 0.005 & & 0.003 & & 0.16 & & 0.03 \\
\hline Sin participación del paciente & $4.4(4.2)$ & & $4,1(3.7)$ & & $3.6(4.7)$ & & $3.5(4.0)$ & & $2.2(2.8)$ & \\
\hline Con participación del paciente & $9.6(6.2)$ & & $10.5(5.8)$ & & $10.7(6.5)$ & & $6.1(3.8)$ & & $6.6(5.7)$ & \\
\hline Conocimiento & & 0.01 & & 0.2 & & 0.004 & & 0.36 & & 0.35 \\
\hline Vicio social & $6.3(5.4)$ & & $6.8(5.5)$ & & $5.5(5.9)$ & & $4.1(4.3)$ & & $3.0(3.8)$ & \\
\hline Enfermedad & $2.0(1.3)$ & & $3.0(2.4)$ & & $1.1(1.6)$ & & $2.4(2.4)$ & & $1.7(1.6)$ & \\
\hline
\end{tabular}

Asociación estadísticamente significativa $(p<0.05)$.

La media está expresada en años.

DE: Desviación Estándar

\section{DISCUSIÓN}

La adicción a sustancias psicoactivas presenta entre sus principales sintomas la ausencia de conciencia de enfermedad $^{24,25}$. Esta característica clínica explica porque el adicto no se siente enfermo si no, por el contrario, se considera una persona sana capaz de controlar su consumo. En estas condiciones la participación de la familia cobra un rol de suma importancia en la identificación de la enfermedad y en la búsqueda de la atención médica ${ }^{15}$. Por otro lado, si bien la familia debería ser quien guía al adicto hacia el tratamiento; en la práctica clínica se observa que muchas de éstas, conociendo que su familiar consume alguna sustancia no busca atención médica de manera oportuna sino que sólo lo hacen cuando aparecen consecuencias negativas en la salud del adicto o la ocurrencia de problemas delictivos para buscar una solución. Dado que en la mayoría de casos la búsqueda de la atención médica es promovida por los familiares, en el presente estudio se propuso evaluar las caracteristicas de los familiares y de sus pacientes para ver como se conjugan en el proceso de la búsqueda de atención médica.

Entre las características de los pacientes vemos que la mayoría $(88.50 \%)$ fueron del género masculino, porcentaje que concuerda con los registros epidemiológicos en el Perú ${ }^{7}$ y de Latinoamérica ${ }^{27,28,29}$. El $80.6 \%$ de la población comenzó el consumo de sustancias entre los 10 y 20 años y el punto de alarma está en que 1 de cada 3 lo hace antes de los 15 años, es decir en plena etapa escolar. Como se observa, la adolescencia constituye un factor de riesgo para el consumo de sustancias ${ }^{30}$; por ejemplo DEVIDA muestra que la población entre los 16 y 19 años presenta un riesgo de 2.6 (IC 95\%: 2.3-3.0) y los de 20 a 24 años de 1.8 (IC 95\%: 1.6-2.1) ${ }^{31}$.

El tiempo transcurrido desde que una persona empieza a consumir la sustancia hasta que llega a la tención médica por primera vez (TC) puede variar entre 7.3 a 14 años depen- diendo del tipo de sustancia. Estudios internacionales muestran que los pacientes pueden demorar 10 años desde que se instaura la dependencia hasta que buscan atención médica ${ }^{5}$, valores similares se observan en la población Mexicana ${ }^{26}$. En Estados Unidos se observó que en los pacientes con abuso de alguna droga demoran en promedio 6 años y los que hacian abuso del alcohol 9 años ${ }^{17}$. En China, la demora en hacer contacto con los servicios de salud es de 17 años $^{32}$. Estos lapsos de demora pueden ser mayores o menores en comparación a otros trastornos (Depresión y Ansiedad) y según el lugar de la evaluación ${ }^{17,26,32}$. El promedio de demora en la población estudiada es mayor al observado en otros paises desarrollados y en vías de desarrollo, sin embargo no llega a ser tan alto como en China. Este prolongado TC puede ser el reflejo de la ausencia de conciencia de enfermedad ${ }^{24,25}$ y a la no percepción de la necesidad de tratamiento que describen estos pacientes ${ }^{33}$. La falta de suficientes servicios de atención médica en el campo de las adicciones también contribuye a la baja la captación de este tipo de pacientes ${ }^{4}$. Una diferencia metodológica con los anteriores trabajos, es que nosotros consideramos como primera atención a la brindada por un psiquiatra, la cual fue realizada por el $21 \%$ de la población, mientras que el porcentaje restante asistió primero a otro tipo de atención. Todo ello muestra que los pacientes acuden a otras instituciones antes de asistir a la atención especializada, conducta que también ha sido observada en Norteamérica donde es más probable que los pacientes acudan primero a psicólogos, religiosos o grupos de auto ayuda que a la atención psiquiátrica ${ }^{5}$.

El mayor TC observado en los consumidores de sustancias legales es explicado por la aceptación social de la que goza el consumo de estas sustancias evidenciada en que la invitación de consumir alcohol por primera vez es hecha por un familiar en el $21 \%$ de casos y que 8 de cada 9 personas a quienes se le invita a consumir tabaco acepta la proposición ${ }^{34}$. Un menor TC es observado en las sustancias ilegales (PBC, cocaina y mari- 
huana) en comparación a las legales, lo cual podría asociarse a que estas sustancias producen sintomas y cambios de conducta más intensos, asi como signos de dependencia de forma más frecuente ${ }^{7}$, lo cual provoca una reacción más temprana hacia la búsqueda de atención médica.

EI TSAM puede variar entre 2.7 y 8.4 años, dependiendo de la sustancia, sin embargo no hemos encontrado estudios que analicen esta variable que nos permita establecer congruencias. Este tiempo prolongado pone de manifiesto la conducta pasiva hacia la búsqueda de atención médica, que puede ser producto de la manipulación a que son sometidos los familiares ${ }^{25}$ y a la condición de coadicción que desarrollan muchos de estos ya que orientan todas sus actividades a la protección del adicto incluso excusándolos de sus responsabilidades ${ }^{10}$. Las sustancias legales también presentan un mayor TSAM comparada a las ilegales, a excepción de la PBC. Esto guarda relación a lo mencionado anteriormente, que por ser las sustancias legales socialmente aceptadas, los familiares no buscan atención médica hasta que producen sintomas severos o se complican con el consumo de sustancias ilegales. EI TSAM para la PBC es mayor incluso a las sustancias legales, lo cual se asocia a lo referido por Nizama, quién encuentra que el $85.5 \%$ de pacientes consumidores de PBC presentaban ausencia de conciencia de enfermedad, negación irreductible de la conducta adictiva y minimización del consumo, asi como manipulación del entorno familiar ${ }^{25}$; todo lo cual ocasiona que los padres, conociendo el problema adictivo, demoren más tiempo en buscar atención médica.

El género resulta un factor importante en la búsqueda de atención médica, y se ha observado que las diferencias intergénero influyen en la búsqueda de atención médica ${ }^{20}$. En nuestro trabajo se observa que las mujeres consumidoras de alcohol llegan más pronto a los servicios de salud en comparación con los varones; sin embargo, este tiempo es de 8.45 años lo cual no deja de ser prolongado. Entre algunas barreras que se han identificado para que las pacientes de sexo femenino no accedan a la atención médica se encuentra la estigmatización, la respuesta del entorno y factores intrínsecos al tratamiento ${ }^{20}$. Otros estudios han determinado las barreras al tratamiento dependiendo del sexo ${ }^{35}$.

La decisión de asistir a la atención médica se encontró asociada al TC y al TSAM en el caso de todas las sustancias evaluadas. La importancia radica en que cuando se toma la decisión de asistir a la atención médica de forma autónoma, se reduce el TC y por tanto las consecuencias del consumo crónico de drogas; además resulta difícil que un paciente con ausencia de conciencia de enfermedad y minimización del consumo acepte que está enfermo. Este hecho se refleja en la práctica clínica, pues muchos de los ingresos hospitalarios se realizan bajo medidas coercitivas. Nizama encontró que el $85.7 \%$ de los pacientes carecian de motivación para recibir tratamiento y sólo una baja proporción evidenció motivación para aceptar ayuda médica ${ }^{25}$. Desde el punto de vista de la entrevista motivacional, estos pacientes tendrian que llegar a la fase de Determinación para que decidan asistir a la atención médica; sin embargo, por el TC tan elevado podemos decir que la mayoría se encuentra dentro de la fase de Precontemplación y Contemplación ${ }^{36,37}$. Otra posible explicación, es que los pacientes no utilizan los servicios de salud, pues no consideran que el uso de sustancias sea una enfermedad y recién acceden a estos cuando observan que no pueden controlar su consumo ${ }^{23}$.

El conocimiento es un factor importante en el proceso de búsqueda de atención médica. En nuestro estudio el 83\% de familiares percibe la adicción como un "vicio social", esta alta proporción se asocia a concepciones que han sido descritas en los familiares, tales como atribuirlos a actos típicos de la edad o a patrones de conducta patrocinados por ciertos grupos sociales ${ }^{34}$, estas mismas concepciones han sido descritos en la población mexicana ${ }^{23}$. Aunque esta variable no pudo ser evaluada en los pacientes, señalamos que Nuño-Gutiérrez menciona que los padres e hijos comparten los mismos conocimientos acerca de las adicciones. Varios estudios apuntan que la concepción del consumo de sustancias como un "vicio social" obstaculiza el uso de los servicios de salud ${ }^{23,37}$. Así mismo, Nuño-Gutiérrez observó que los pacientes consumidores de sustancias y sus padres consideraban, al inicio del consumo, el uso de sustancias como un vicio donde destaca la voluntad del sujeto en su inicio y por tanto también en su cese; una vez que el usuario experimentaba problemas por el consumo, redefinian el problema como una enfermedad, y es entonces donde recién hacen uso de los servicios de salud ${ }^{23}$. En los casos de las sustancias donde no se evidencia una diferencia significativa en la asociación entre conocimiento y $\mathrm{TC}$, también se llega a observar que en promedio, aquellos que conciben a la adicción como "vicio social" demoran más en llegar a la atención médica.

El TC y el TSAM evalúan las conductas en la búsqueda de atención médica por parte de los pacientes y familiares, respectivamente; y al ser tan prolongadas y guardar entre si una relación directamente proporcional, nos muestra que la falta de decisión de los familiares en acudir a los centros de atención médica, provoca o contribuye a que sus parientes continúen consumiendo sustancias y por lo tanto se facilite la producción de los efectos deletéreos del consumo crónico. La diferencia entre el TC y el TSAM representa el tiempo desde que los pacientes inician el consumo hasta que los familiares lo perciben y puede variar de 4.6 a 8.6 años. Al ser prolongado nos muestra que existe una fase dentro del proceso adictivo donde el adicto puede esconder el consumo de su entorno social y sólo manifestarse indirectamente en problemas como fracaso escolar, mal desempeño laboral ${ }^{39}$, embarazo a temprana edad ${ }^{40}$ y violencia e inestabilidad familiar ${ }^{8,9}$.

Una limitación del estudio fue la metódica por la cual se obtuvo el TC, la cual se realizó a través de una entrevista con los familiares; y no como hubiera sido ideal obtenerlo directamente de los pacientes. Por ello consideramos la posibilidad de una subestimación de la cifra real; sin embargo, el haber trabajado con los familiares nos permite analizar el otro componente importante en la búsqueda de atención médica, ya que consideramos parte fundamental a la familia en la identificación de la enfermedad y en el mantenimiento del tratamiento. Además, hasta donde tenemos información este trabajo constituye el primer informe presentado a la comunidad médica acerca de la influencia que puede dar el entorno familiar en la búsqueda de atención médica. 
En el Perú, por el actual número de consumidores existentes, se debería actuar con mayor énfasis a nivel de la prevención secundaria, buscando mecanismos que promuevan que los pacientes consumidores de sustancias psicoactivas hagan uso de los servicios de salud. Según los resultados obtenidos en el trabajo, recomendamos que las campañas preventivo promocionales deben estar dirigidas a los grupos de alto riesgo y familiares, combatiendo los conceptos erróneos que existen en ambos grupos en torno a las adicciones como "vicio social". Además debería incidirse en el concepto de adicción como enfermedad, pues esto genera una búsqueda más temprana de atención médica. También se debe sugerir que una vez identificado el problema, la familia no debiera esperar la participación del paciente para asistir a la atención médica, sino tomar la decisión de manera autónoma.

\section{AGRADECIMIENTOS}

Se brinda el respectivo agradecimiento a los Drs. Martín Nizama Valladolid y César Gutiérrez Villafuerte y a la Dra. Elisa del Rosario Salvador Villacorta, por la colaboración y apoyo en la realización del trabajo.

\section{REFERENCIAS}

1. Naciones Unidas. Tendencias Mundiales de las Drogas llícitas 2003. New York: Oficina contra las Drogas y el Delito; 2003.

2. United Nations. World Drug Report 2007. Slovakia: Office on drugs and crime; 2007.

3. Devida. Información Estadisticas sobre Drogas en el Perú. Lima: Observatorio peruano de drogas - OPD; 2006.

4. Naciones Unidas. Drogas y delitos en el Perú. Situación actual y evolución. Lima: Oficina regional para Perú y Ecuador; 2007.

5. Wang PS, Berglund PA, Olfson M, Kessler RC. Delay in initial treatment contact after first onset of a mental disorder. Health Serv Res 2004; 39: 393-415.

6. Ministerio de Salud. Plan Nacional Concertado de Salud. Lima: Ministerio de Salud; 2007.

7. Devida. III Encuesta Nacional de consumo de Drogas en Población General de Perú 2006. Lima: Devida; 2007.

8. Gelder M, Gath D, Mayou R. Concise Oxford Textbook of Psychiatric. Oxford: Oxford University Press 1994.

9. Barrett A, Turner J. Family structure and substance use problems in adolescence and early adulthood: examining explanations for the relationship. Addiction 2006; 101: 109-20.

10. Jiménez J. Familia, Trastorno Adictivo y Codependencia. Revista de Psiquiatría y Salud Mental Hermilio Valdizán [En línea] JulioDiciembre 2002; 3(2): [aprox.4p.]. Disponible en: http://www. minsa.gob.pe/hhv/revista/2002I//indice.htm[Citado el 12 de Diciembre del 2008]

11. Copello A, Orford J. Addiction and the family: is it time for services to take notice of the evidence? Addiction 2002; 97: 1361-3.

12. Englund MM, Egeland B, Oliva EM, Collins WA. Childhood and adolescent predictors of heavy drinking and alcohol use disorders in early adulthood: a longitudinal developmental analysis. Addiction 2008; 103 suppl. 1: 23-35.
13. Merline A, Jager J, Schulenberg JE. Adolescent risk factors for adult alcohol use and abuse: stability and change of predictive value across early and middle adulthood. Addiction 2008; 103 suppl. 1: 84-99.

14. Simons L, Giorgio T. Characteristics of Substance Abusing Men and Women entering a Drug Treatment Program. Addictive Disorders \& Their Treatment 2008; 7: 15-23.

15. Saatcioglu O, Erim R, Cakmak D. Role of family in alcohol and substance abuse. Psychiatry Clin Neurosci 2006; 60: 125-132.

16. Usher $K_{1}$ Jackson D, O'Brien L. Adolescent drug abuse: Helping families survive. Int J Ment Health Nurs 2005; 14: 209-214.

17. Wang PS, Berglund P, Olfson M, Pincus HA, Wells KB, Kessler RC. Failure and delay in initial treatment contact after first onset of mental disorders in the National Comorbidity Survey replication. Arch Gen Psychiatry 2005; 62: 603-613.

18. Wenzel SL, Burnam MA, Koegel P, Morton SC, Miu A, Jinnett $\mathrm{KJ}$, et al. Access to inpatient or residential substance abuse treatment among homeless adults with alcohol or other drug use disorders. Med Care 2001; 39: 1158-69.

19. Fortney JC, Blow FC, Bunn JY. The effects of travel barriers and age on the utilization of alcoholism treatment aftercare. Am J Drug Alcohol Abuse 1995; 21: 391-406.

20. Tomás-Dols, S.; Valderrama-Zurián, J. C.; Vidal-Infer, A.; SamperGras, T.; Hernández-Martínez, Ma C.; Torrijo-Rodrigo, Ma J. Género y barreras de accesibilidad al tratamiento en pacientes adictos al alcohol de la Comunidad Valenciana. Adicciones 2007; 19:169-178.

21. Marion TR, Stefanik-Campisi C. The elderly alcoholic: identification of factors that influence the giving and receiving of help. Perspect Psychiatr Care 1990; 25: 32-5.

22. Desai MM, Rosenheck RA, Craig TJ. Screening for alcohol use disorders among medical outpatients: the influence of individual and facility characteristics. Am J Psychiatry 2005; 162: 1521-6.

23. Nuño-Gutiérrez BL, Álvarez-Nemegyei J, González-Fortaleza C, Madrigal-de León EA. La adicción ¿Vicio o Enfermedad? Imágenes y uso de los servicios de salud en adolescentes usuarios y sus padres. Salud Mental 2006; 29: 47-54.

24. Nizama M. Guía para el manejo familiar de las adicciones. Lima: Ed. Universidad Alas Peruanas; 2003.

25. Nizama M. Estudio fenomenológico descriptivo de 120 pacientes dependientes principalmente a pasta básica de cocaína. [Tesis Doctoral]. Lima: Universidad Nacional Mayor de San Marcos; 1991.

26. Borges G, Wang PS, Medina-Mora ME, Lara C, Chiu WT. Delay of first treatment of mental and substance use disorders in Mexico. Am J Public Health 2007; 97: 1638-43.

27. Conadic. Encuesta Nacional de adicciones 2002. México: Conadic; 2003.

28. Miguez H. Consumo de sustancias psicoactivas en Argentina. Psicoactiva 2000; 18: 1-17.

29. Fuentealba R. Cumsille F, Araneda J. Consumo de drogas lícitas e ilícitas en Chile: Resultados del estudio de 1998 y comparación con los estudios de 1994 y 1996. Rev Panam Salud Pública 2000; 7: 79- 87.

30. Ramírez $M$, Andrade D. La Familia y los factores de riesgo relacionados con el consumo de alcohol y tabaco en los niños y adolescentes (Guayaquil-Ecuador). Rev Latino-Am Enfermagen 2005; 13: 813-18. 
31. Devida. II Encuesta Nacional sobre consumo y prevención de drogas 2002. Lima: Devida; 2003.

32. Lee $S$, Fung SC, Tsang A, Zhang MY, Huang YQ, He YL, et al. Delay in initial treatment contact after first onset of mental disorders in metropolitan China. Acta Psychiatr Scand 2007; 116: 10-16.

33. Mojtabai $R$, Olfson M, Mechanic D. Perceived need and helpseeking in adults with mood, anxiety, or substance use disorders. Arch Gen Psychiatry 2002; 59: 79-84.

34. Cedro. Epidemiología de las drogas en la población peruana 2005, encuesta de hogares. Lima: Cedro; 2006.

35. Brady TM, Ashley OS, editores. Women in substance abuse treatment: results from the Alcohol and Drug Services Study (ADSS). Rockville, MD: Substance Abuse and Mental Health Service Administration; 2005.
36. Lizárraga $S$, Ayarra M. Entrevista motivacional. Anales Sis San Navarra 2001; 4 supl. 2: 41-48.

37. Fuentes-Pila J, Calatayud P, López E, Castañeda B. La entrevista motivacional: llave del proceso de cambio en la dependencia nicotín-tabáquica. Trastornos Adictivos 2005; 7: 153-65.

38. Souza y Machorro M. Mitología desinformación e ignorancia en adicciones. Liberaddictus 2003; 74: 2-7.

39. Villanueva $M$, Factores psicológicos asociados a la iniciación y habituación. En: León F, Castro de la Mata R. Pasta básica de cocaina, un estudio multidisciplinario. Lima: Cedro, 1989. p. 167-209.

40. Forthofer MS, Kessler RC, Story AL, Gotlib IH. The effects of psychiatric disorders on the probability and timing of first marriage. J Health Soc Behav 1996; 37:121-132. 